\title{
Chromosomes in childhood acute lymphoblastic leukaemia: Karyotypic patterns in disease subtypes
}

\author{
M A Wodzinski, A E Watmore, J S Lilleyman, A M Potter
}

\begin{abstract}
To define further the clinical importance of cytogenetic analysis in acute lymphoblastic leukaemia (ALL) a prospective study was performed on 139 unselected children. Analyses were considered adequate in 104, of whom 35 were normal and 69 had clonal abnormalities. Abnormalities were categorised according to banded chromosome analysis as well as chromosome count. Karyotypes were correlated with clinical and laboratory features at diagnosis and with survival. Of the successful analyses, thirty five (34\%) children had no abnormalities; this group contained an excess of $\mathrm{T}$ cell disease. Twenty five $(24 \%)$ had a "characteristic" hyperdiploid karyotype and as a group had lower presenting white counts, a tendency to $\mathrm{CD} 10$, and periodic acid schiff positivity of the blast cells and smaller spleens. None was an infant and only one was over 10 years old. Seven $(7 \%)$ children with $t(9 ; 22)$, $t(8 ; 14)$, or $t(4 ; 11)$ translocations were grouped together as "specific" translocations. Collectively they had a significantly worse prognosis than the remainder. Nine children developed central nervous system relapse, six of whom had either $t(4 ; 11)$ or abnormalities of $9 p$ or $19 p$.

A descriptive classification taking into account chromosome bonding pattern is cytogenetically more appropriate and may be more clinically useful than grouping children simply by chromosome number. As knowledge and techniques improve, the classification of cytogenetic abnormalities in ALL will need to be kept under frequent review.
\end{abstract}

Department of Haematology, The Children's Hospital, Sheffield S10 2TH. M A Wodzinski J S Lilleyman Centre for Human Genetics, Lang Hill, Manchester Road, Sheffield S10 2DN A E Watmore A M Potter

Correspondence to: Dr M A Wodzinski, Department of $H$ aematology, Department of Haematology, Northern General Hospital S5 7AU.

Accepted for publication 29 August 1990
Cytogenetic analysis of blast cells in childhood acute lymphoblastic leukaemia (ALL) has become increasingly successful and refined in recent years and is thought to contribute clinically important information. ${ }^{1}$ To explore this point further we undertook an analysis of consecutive patients presenting over a period of 11 years. The cytogenetic findings were correlated with clinical and laboratory features at presentation, and against the outcome of treatment. An attempt was made to take into account chromosome banding pattern as well as simply using chromosome number which has been a common approach in the past. ${ }^{1}$

\section{Methods}

Bone marrow samples were available for chromosome analysis from 139 of 148 consecutive children who presented with acute lymphoblastic leukaemia (ALL) between January 1979 and February 1990 at the Sheffield Children's Hospital. Chromosome analysis was performed on direct preparations or short term cultures. Banding was by the trypsin/Giemsa method. Analysis was considered successful if two or more cells were seen with the same chromosomal abnormality, two or more cells were seen with more than 50 chromosomes, or in the case of normal results 10 banded cells were seen without clonal abnormality.

\section{CLASSIFICATION}

Abnormal results were grouped as follows, using a combination of chromosome banding and chromosome number:

Unbanded abnormalities ( $U A$ ): This category included patients with unidentifiable chromosome abnormalities with a count of 50 or less.

"Characteristic" hyperdiploidy ( $\mathrm{CH})$ : The conventional definition of high hyperdiploidy ${ }^{1}$ was extended to include cases with 48-50 chromosomes if banding showed extra chromosomes typical of the $>50$ group. Cases with chromosome counts in the tetraploid range were not included.

"Specific" translocations (ST): This category included patients with the recognised and relatively common translocations $\mathrm{t}(9 ; 22), \mathrm{t}(4$; $11)$, and $t(8 ; 14)$ and their variants. These have been noted to be associated with high risk disease, ${ }^{1}$ and were therefore considered separately from other rearrangements.

Near tetraploidy (NT): Patients with chromosome counts in the near tetraploid range were separated from those with the characteristic pattern of hyperdiploidy.

Other abnormalities $(O A)$ : This group comprised the remaining patients with chromosomal abnormalities that did not fall into the above categories. It was a cytogenetically heterogeneous group, but there was no logical way to subdivide the patients into subgroups.

The chromosomal findings were related to the following features at presentation: age; sex; haemoglobin; white cell count; platelet count; splenomegaly; hepatomegaly; lymphadenopathy; central nervous system (CNS) disease; French-American-British (FAB) subclass $^{2}$; immunological phenotype; and periodic acid Schiff (PAS) positivity of the blasts. The 
Table 1 Distribution of karyotypes according to year of diagnosis

\begin{tabular}{|c|c|c|c|}
\hline Karyotype & $1979-1982$ & $1983-1986$ & $1987-1990$ \\
\hline $\begin{array}{l}\text { Normal } \\
\text { Unbanded abnormalities } \\
\text { Characteristic hyperdiploidy } \\
\text { Specific translocations } \\
\text { Near tetraploidy } \\
\text { Other abnormalities } \\
\text { Failed } \\
\text { Total }\end{array}$ & $\begin{array}{rr}8 & (36 \%) \\
6 & (27 \%) \\
1 & (59 \%) \\
2 & (9 \%) \\
1 & (5 \%) \\
4 & (18 \%) \\
23 & \\
45 & \end{array}$ & $\begin{aligned} 17 & (35 \%) \\
5 & (10 \%) \\
13 & (27 \%) \\
3 & (6 \%) \\
1 & (2 \%) \\
9 & (19 \%) \\
8 & \\
56 & \end{aligned}$ & $\begin{array}{rr}10 & (29 \%) \\
0 & (0 \%) \\
11 & (32 \%) \\
2 & (6 \%) \\
1 & (3 \%) \\
10 & (29 \%) \\
4 & \\
38 & \end{array}$ \\
\hline
\end{tabular}

Figures in parentheses represent the percentage of successful analyses for the given period.

morphological (FAB) subclass was determined by three independent observers. Cases were considered positive for CD10 if $30 \%$ or more, and for PAS, if $10 \%$ or more blasts were positive. Most of the children who typed as CD10 positive had cytoplasmic immunoglobulin determined (CIg) and were thus subclassified as early pre-B or pre-B ALL.

Most children were entered into the eighth or tenth Medical Research Council's United Kingdom ALL trials, though in the first two years children were entered into earlier trials. Survival data were calculated as at 31.5.90.

Contingency table analysis with $\chi^{2}$ tests and, where appropriate, Yates' continuity correction were used. Event free survival was calculated for each group of patients and differences between groups were analysed according to the log-rank method. ${ }^{3}$ The median follow up was 68 months. Failure to achieve remission or relapse at any site were counted as adverse events. Deaths in remission were censored at the time of death.

\section{Results}

Cytogenetic analysis was attempted on 139 children and was successful in 104. Clonal abnormalities were found in 69 children and no abnormality was detected in 35 despite analysis of a minimum of 10 banded cells. The results, summarised in table 1 , have been subdivided according to date of diagnosis to illustrate certain differences of distribution. Most of the
35 failures occurred in the first four years of the study. Few cases of "characteristic" hyperdiploidy were detected then. Unbanded abnormalities comprised $27 \%$ of successful analyses in this period, whereas recently all have been banded.

The presenting features and disease outcome of patients with successful analyses are summarised in table 2 . The detailed karyotypes and main disease characteristics of the "specific" translocations group and the other abnormalities group are given in table 3 .

Abnormal karyotypes were detected but not characterised by banding in 11 cases. Of the remaining clonal abnormalities identified by banding, 25 were "characteristic" hyperdiploidy, seven were "specific" translocations, three were near tetraploid and 23 were other abnormalities.

There was a significant excess of $T$ cell disease among patients with normal karyotypes compared with those with a clonal abnormality (11/35 vs 5/69, $\chi^{2}=8.65, \mathrm{p}=<0.01$ ).

The "characteristic" hyperdiploidy group, when compared with the other analysable cases, had a lower white cell count (for WCC $<20 \times 10^{9} / 1, \chi^{2}=4.9, \mathrm{p}=<0.05$ ), a tendency to periodic acid Schiff and CD10 positivity of the blast cells $\left(\chi^{2}=5 \cdot 1\right.$ and $4 \cdot 4$, respectively, $p$ $=<0.05)$, a greater proportion of children aged $2-12$ y $\left(\chi^{2}=4.89, \mathrm{p}=<0.05\right)$, and more with minimal or no splenomegaly $(2 / 25$ vs $28 /$ $\left.79, \chi^{2}=5 \cdot 7, \mathrm{p}=<0 \cdot 02\right)$. Although there was a trend towards longer remission, it did not reach significance $\left(\log\right.$ rank $\left.\chi^{2}=3 \cdot 6, p=0.06\right)$. The ratio of early pre-B to pre-B phenotypes in this group (18:4), although high, was not significantly different when compared with other groups (29:20); $\chi^{2}=2 \cdot 5, p=0 \cdot 15$. Eight children with "characteristic" hyperdiploidy also had structural chromosomal abnormalities, eight did not, and in the remaining nine cases the preparations were not of sufficient quality to determine the point. Of three children with "characteristic" hyperdiploidy who relapsed, two had no detectable structural

Table 2 Summary of presenting features and outcome by chromosomal category

\begin{tabular}{|c|c|c|c|c|c|c|c|}
\hline \multirow[b]{2}{*}{ Clinical and laboratory findings } & \multirow[b]{2}{*}{ Total } & \multirow{2}{*}{$\begin{array}{l}\text { Normal } \\
\text { karyotype }\end{array}$} & \multicolumn{5}{|c|}{ Abnormal karyotypes } \\
\hline & & & $U A$ & $\mathrm{CH}$ & $S T$ & $N T$ & $O A$ \\
\hline Total No of cases & 104 & 35 & 11 & 25 & 7 & 3 & 23 \\
\hline Sex: Male & 62 & 23 & 7 & 14 & 4 & 2 & 12 \\
\hline Female & 42 & 12 & 4 & 11 & 3 & $\overline{1}$ & 11 \\
\hline Age: $<1$ & 7 & 2 & 0 & 0 & 2 & 0 & 3 \\
\hline $1-$ & 28 & 14 & 1 & 8 & 1 & 0 & 4 \\
\hline $3-$ & 40 & 12 & 6 & 12 & 2 & 1 & 7 \\
\hline $7-$ & 16 & 5 & 2 & 4 & 1 & 1 & 3 \\
\hline $10-$ & 13 & 2 & 2 & 1 & 1 & 1 & 6 \\
\hline Mean age & $8 \cdot 0$ & $4 \cdot 2$ & $6 \cdot 3$ & 4.9 & $4 \cdot 7$ & $7 \cdot 7$ & 5.9 \\
\hline WCC: $<20 \times 10^{9} / 1$ & 57 & 17 & 5 & 20 & 2 & 2 & 11 \\
\hline $20-50 \times 10^{9} / 1$ & 16 & 5 & 2 & 3 & 2 & 0 & 4 \\
\hline$>50 \times 10^{9} / 1$ & 31 & 13 & 4 & 2 & 3 & 1 & 8 \\
\hline FAB type: L1 & 93 & 32 & 10 & 24 & 4 & 3 & 20 \\
\hline L2 & 9 & 3 & 1 & 1 & i & 0 & 3 \\
\hline L3 & 2 & 0 & 0 & 0 & 2 & 0 & 0 \\
\hline Phenotype: c-ALL & 76 & 21 & 9 & 25 & 4 & 2 & 15 \\
\hline null-ALL & 8 & 2 & 1 & 0 & 2 & 0 & 3 \\
\hline T-ALL & 16 & 11 & $i$ & 0 & 0 & 1 & 3 \\
\hline B-ALL & 1 & 0 & 0 & 0 & 1 & 0 & 0 \\
\hline ? & $i$ & 1 & 0 & 0 & 0 & 0 & 2 \\
\hline Outcome: Induction failure & 5 & 3 & 0 & 0 & 1 & 1 & 0 \\
\hline Relapse & 19 & 10 & 1 & 3 & 4 & 1 & 6 \\
\hline
\end{tabular}

Key: UA = Unbanded abnormalities; $\mathrm{CH}=$ Characteristic hyperdiploidy; $\mathrm{ST}=$ Specific translocations; NT = Near tetraploidy; $\mathrm{OA}=$ Other abnormalities; $\mathrm{WCC}=$ White cell count at presentation; FAB $=$ French-American-British classification. 
Table 3 Karyotype and clinical features of specific translocation and other abnormalities groups

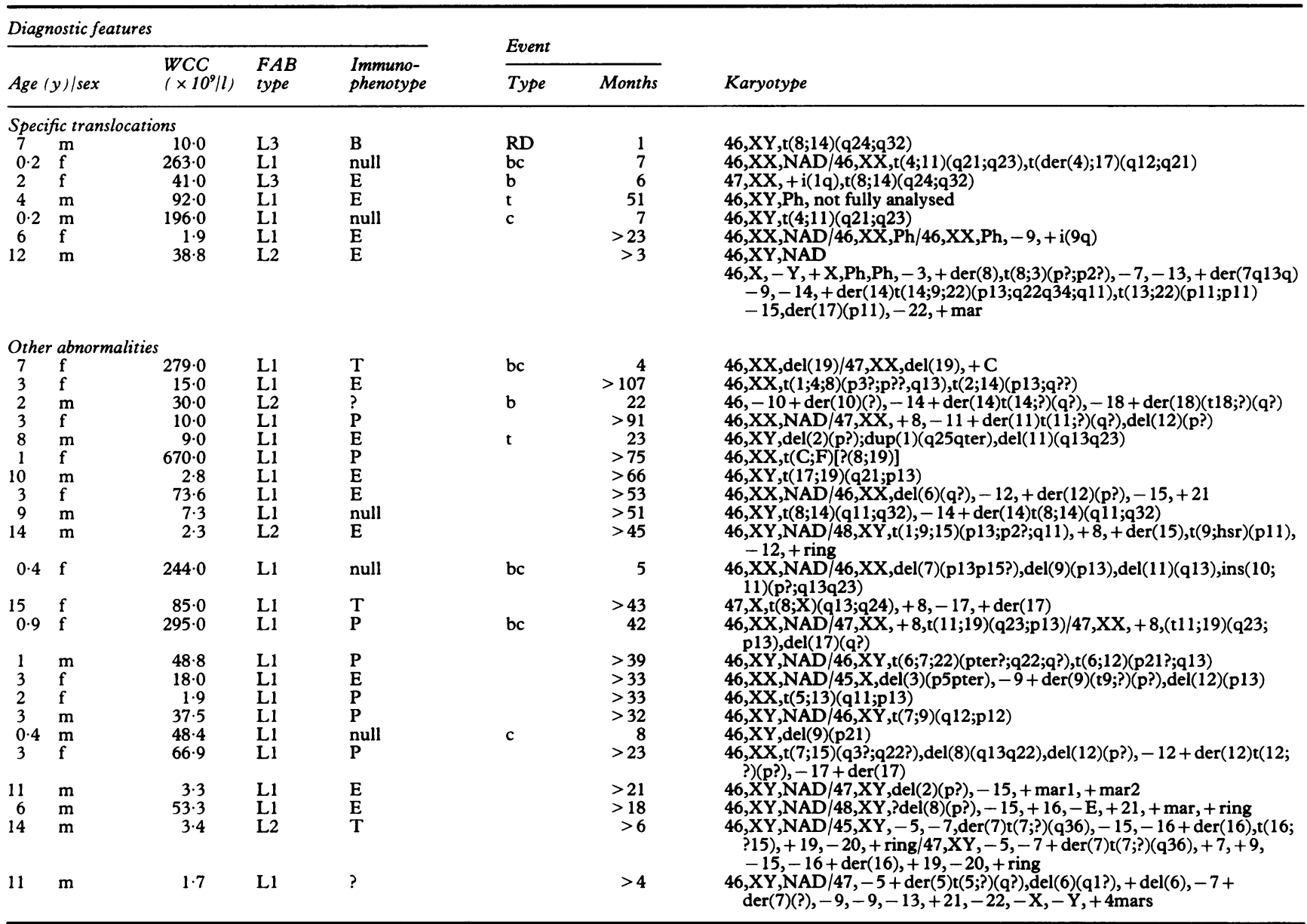

Key: Immunophenotype: "C" common (cytoplasmic $\mu$ not determined). $\mathrm{E}=$ early pre-B subcategory of common; $\mathrm{P}=$ pre-B subcategory of common; $\mathrm{T}=\mathrm{T}$-cell; $\mathrm{B}=\mathbf{B}$-cell.

Events-type: $b=$ bone marrow relapse; $c=C N S$ relapse; $b c=$ bone marrow and CNS relapse; $t=$ testicular relapse; $R D=$ resistant disease

Time from diagnosis to event is given in months.

abnormality, and in one the position was undetermined.

The seven children with "specific" translocations included the only two cases of L3 leukaemias and, as a group, they had a worse prognosis than the other banded abnormal cases (log rank $\left.\chi^{2}=8 \cdot 7, p=<0 \cdot 01\right)$, or the remaining analysable cases $\left(\log \operatorname{rank} \chi^{2}=7 \cdot 82\right.$, $\mathrm{p}=<0.01)$. There was no significant difference in leukaemia free survival between any other cytogenetic groups, although when the nine cases with CNS relapse were considered, certain chromosome changes were overrepresented. These were $t(4 ; 11)$ (both cases), abnormalities of $9 p$ (two of six cases, both simple deletions), and abnormalities of $19 \mathrm{p}$ (two of four cases). The remaining three CNS relapses occurred in one case of hyperdiploidy and two cases with a normal karyotype. The presenting white cell count of these nine cases was between $48.4 \times 10^{9} / 1$ and $295.0 \times 10^{9} / 1$, with a median of $196.0 \times 10^{9} / 1$.

\section{Discussion}

A major consideration when classifying children with ALL according to their karyotype is the technical improvement in cytogenetic techniques which has occurred over the past decade. Over 11 years there has been a declining failure rate in our patients and an increase in the number of abnormalities detected, leading to far more accurate chromosome analyses in the latter part of the study. Follow up on these cases has of course been short. Despite this difficulty, some tentative conclusions can be drawn.

The striking feature of the 35 children in whom no clonal abnormality was detected was the excess of children with $\mathrm{T}$ cell disease -11 of $35(31 \%), \chi^{2}=8 \cdot 4, p=<0.01$. This confirms the results of earlier work which also showed that an excess of children with normal karyotype-19 of $38(50 \%)$, had T cell disease, ${ }^{4}$ but it has been suggested that virtually all cases of ALL have clonal abnormalities if analyses are sufficiently detailed. ${ }^{4}$

Although 22 of our patients had a pre-B immunophenotype, no case of the supposedly associated $^{5} t(1 ; 19)$ translocation was observed. In one study that translocation occurred in 19 of $79(24 \%)$ such children, ${ }^{4}$ but in another study of 131 children with all types of ALL no examples were found, though the incidence of immunologically defined pre-B disease was not given. ${ }^{6}$ It may be that the incidence of $t(1 ; 19)$ is not as high as has been suggested, and its association with pre-B ALL may be less clearcut than supposed.

It has been claimed that the presence of any translocation confers a sixfold greater risk of early treatment failure compared with absence of translocation, ${ }^{7}$ and that patients with the Philadelphia chromosome, $t(8 ; 14)$ and $t(4 ; 11)$ have a worse prognosis than those with other 
translocations. ${ }^{1}$ Although we were able to confirm that these "specific" translocations as a group had a worse prognosis than patients from the other abnormalities group, we were unable to detect any difference in prognosis between the latter group, all of whom had structural rearrangements, and the remaining cytogenetic categories.

We defined "characteristic" hyperdiploidy to include patients with $48-50$ chromosomes if banding showed only chromosomes typical of the $>50$ group, and to exclude near tetraploidy. Of the three patients with near tetraploidy, one had a very high presenting white cell count and a $\mathrm{T}$ cell phenotype. Pui et al found a 3\% incidence of $\mathrm{T}$ cell disease in their patients with high hyperdiploidy, but the chromosomal number was not stated. ${ }^{8}$ They too found only three cases of near tetraploidy, so small numbers prevent further analysis of this rare subgroup.

Only two $(2 \%)$ of our patients had a hypodiploid clone. This is a lower incidence than the $7 \%$ found by Pui et al. ${ }^{9}$ Neither had less than 45 chromosomes or a near haploid karyotype, both of which have been described in a substantial proportion of hypodiploid patients. ${ }^{10}$ Both were mosaics with a 45 chromosome clone combined with a normal clone, and one also had a 47 chromosome clone.

Apart from the apparently poor prognosis of our special translocation patients, we were unable to show any survival differences between the other chromosome groups, though our numbers are necessarily relatively small. A recent study of a similar sized group also failed to show survival differences between the conventional cytogenetic groups, ${ }^{6}$ but others have suggested that high hyperdiploidy confers an advantage, ${ }^{41-15}$ whereas hypodiploidy bodes ill. ${ }^{9}$ These discrepancies may partly be due to small numbers and incomplete follow up, though it is at least possible that the prognostic importance of cytogenetic features could be modified by improvements in treatment. ${ }^{6}$

There did seem to be an increased risk of CNS relapse with certain karyotypes. This occurred in both of the two $t(4 ; 11)$ children, two of the six with abnormalities of $9 p$, and two of the four with abnormalities of $19 \mathrm{p}$. It has been noted that $t(4 ; 11)$ carries a high risk of relapse at any site, ${ }^{1}$ and it has recently been claimed that abnormalities of the short arm of chromosome 9 confer a high risk of CNS relapse, especially where material is deleted. ${ }^{16}$ Our data would support this. Both relapses in our 9p group had a simple deletion. Abnormalities of $19 p$ in general have not been noted to carry a particular risk of CNS relapse, though it has recently been claimed that $t(11$; 19) has a high incidence of CNS being affected at presentation and a high risk of relapse. ${ }^{17}$ Our sole child with that translocation did not have CNS disease at presentation, but has relapsed in both marrow and CNS.

The practice of categorising chromosomal abnormalities by using simple chromosome number predates banding. With banded karyotypes these categories should be reassessed, incorporating more precise chromosome abnormality groupings to increase their clinical usefulness. Our redefined "characteristic" hyperdiploid group, when compared with the other analysable cases, showed less striking splenomegaly and a predilection towards the 2-12 year age group. These features did not reach significance if the three near tetraploidy cases were included and the hyperdiploid cases with 50 chromosomes or less were excluded in accordance with previous classifications. The trend to longer survival was also more suggestive using our modified definition.

What can be concluded from all this? Cytogenetic analysis in ALL is undeniably very labour intensive and expensive, and its clinical importance is presently limited to the detection of clonal abnormalities in the few marrows where the diagnosis of leukaemia is in doubt, and to the prediction of outcome in a very small group of children with specific abnormalities such as those of our specific translocation group. On the other hand, it could be argued that current techniques, which may improve further, have not been applied long enough to large enough numbers for the true value of cytogenetics to emerge, and that much more work is needed.

1 Bloomfield CD, Secker-Walker LM, Goldman AI, et al Six-year follow-up of the clinical significance of karyotype in acute lymphoblastic leukemia. Cancer Genet Cytogenet 1989;40:171-85.

2 Bennett JM, Catovsky D, Daniel MT, et al. Morphological classification of acute lymphoblastic leukaemia: concordance among observers and clinical correlations. $\mathrm{Br}$ Haematol 1981;47:553-61.

3 Peto R, Pike MC, Armitage P, et al. Design and analysis of randomised clinical trials requiring prolonged observation of each patient. Br J Cancer 1977;35:1-39.

4 Pui C-H, Williams DL, Roberson PK, et al. Correlation of karyotype and immunophenotype in childhood acute lymphoblastic leukemia. J Clin Oncol 1988;6:56-61.

5 Shikano T, Kaneko Y, Takazawa M, Ueno N, Ohkawa M, Fujimoto T. Balanced and unbalanced $1 ; 19$ translocationassociated acute lymphoblastic leukemias. Cancer 1986; 58:2239-43.

6 Fletcher JA, Kimball VM, Lynch E, et al. Prognostic implications of cytogenetic studies in an intensively treated group of children with acute lymphoblastic leukemia. Blood 1989;74:2130-5.

7 Williams DL, Harber J, Murphy SB, et al. Chromosomal translocations play a unique role in influencing prognosis in childhood acute lymphoblastic leukemia. Blood 1986; 68:205-12.

8 Pui C-H, Raimondi SC, Dodge RK, et al. Prognostic importance of structural chromosomal abnormalities in children with hyperdiploid ( $>50$ chromosomes) acute children with hyperdiploid ( $>50$ chromosom
lymphoblastic leukemia. Blood 1989;73:1963-7.

9 Pui C-H, Williams DL, Raimondi SC, et al. Hypodiploidy is associated with a poor prognosis in childhood acute lymphoblastic leukemia. Blood 1987;70:247-53.

10 Pui C-H, Carroll AJ, Raimondi SC, et al. Clinical presentation, karyotypic characterization, and treatment outcome of childhood acute lymphoblastic leukemia with a nearhaploid or hypodiploid < 45 line. Blood 1990;75:1170-7.

11 Fenaux P, Lai JL, Morel P, et al. Cytogenetics and their prognostic value in childhood and adult acute lymphoblastic leukemia (ALL) excluding L. Hematol Oncol 1989 7:307-17.

12 Heinonen $\dot{K}$, Rautonen J, Siimes MA, Knuutila S. Cytogenetic study of 105 children with acute lymphoblastic leukemia. Eur $J$ Haematol 1988,41:237-42.

13 Secker-Walker LM, Chessells JM, Stewart EL, Swansbury GJ, Richards S, Lawler SD. Chromosomes and other prognostic factors in acute lymphoblastic leukaemia: a long-term follow-up. $B r J$ Haematol 1989;72:336-42

14 Michael PM, Garson OM, Ekert H, Tauro G, Rennie GC, Pilkington GR. Prospective study of childhood acute lymphoblastic leukemia: hematologic, immunologic and cytogenetic correlations. Med Pediatr Oncol 1988;16: 153-61.

15 Heim S, Bekassy AN, Garwicz S, et al. Bone marrow karyotypes in 94 children with acute leukaemia. Eur $J$ Haematol 1990;44:227-33.

16 Murphy SB, Raimondi SC, Rivera GK, et al. Nonrandom abnormalities of chromosome $9 p$ in childhood acute lymphoblastic leukemia: association

17 Gibbons B, Katz FE, Ganly P, Chessells JM. Infant acute lymphoblastic leukaemia with $\mathrm{t}(11 ; 19)$. Br J Haematol 1990;74:264-9. 